\title{
Where have all the mosquito nets gone? Spatial modelling reveals mosquito net distributions across Tanzania do not target optimal Anopheles mosquito habitats
}

\author{
Emily S. Acheson*, Andrew A. Plowright and Jeremy T. Kerr
}

\begin{abstract}
Background: Malaria remains the deadliest vector-borne disease despite long-term, costly control efforts. The United Republic of Tanzania has implemented countrywide anti-malarial interventions over more than a decade, including national insecticide-treated net (ITN) rollouts and subsequent monitoring. While previous analyses have compared spatial variation in malaria endemicity with ITN distributions, no study has yet compared Anopheles habitat suitability to determine proper allocation of ITNs. This study assesses where mosquitoes were most likely to thrive before implementation of large-scale ITN interventions in Tanzania and determine if ITN distributions successfully targeted those areas.
\end{abstract}

Methods: Using Maxent, a species distribution model was constructed relating anopheline mosquito occurrences for 1999-2003 to high resolution environmental observations. A 2011-2012 layer of mosquito net ownership was created using georeferenced data across Tanzania from the Demographic and Health Surveys. The baseline mosquito habitat suitability was compared to subsequent ITN ownership using (1) the average ITN numbers per house and (2) the proportion of households with $\geq 1$ net to test whether national ITN ownership targets have been met and have tracked malaria risk.

Results: Elevation, land cover, and human population distribution outperformed variants of temperature and Normalized Difference Vegetation Index (NDVI) in anopheline distribution models. The spatial distribution of ITN ownership across Tanzania was near-random spatially (Moran's I = 0.07). Householders reported owning 2.488 ITNs on average and $93.41 \%$ of households had $\geq 1$ ITN. Mosquito habitat suitability was statistically unrelated to reported ITN ownership and very weakly to the proportion of households with $\geq 1$ ITN $\left(R^{2}=0.051\right)$. Proportional ITN ownership/ household varied relative to mosquito habitat suitability (Levene's test $F=3.0037$ ). Quantile regression was used to assess trends in ITN ownership among households with the highest and lowest $10 \%$ of ITN ownership. ITN ownership declined significantly toward areas with the highest vector habitat suitability among households with lowest ITN ownership ( $\mathrm{t}=-3.38$ ). In areas with lowest habitat suitability, ITN ownership was consistently higher.

Conclusions: Insecticide-treated net ownership is critical for malaria control. While Tanzania-wide efforts to distribute ITNs has reduced malaria impacts, gaps and variance in ITN ownership are unexpectedly large in areas where malaria risk is highest. Supplemental ITN distributions targeting prime Anopheles habitats are likely to have disproportionate human health benefits.

Keywords: Anopheles mosquito, Insecticide-treated nets, ITN, Malaria, Modelling, Niche, Species distribution models, Vector

\footnotetext{
*Correspondence: eache094@uottawa.ca

Department of Biology, University of Ottawa, Gendron 352, 30 Marie

Curie, Ottawa, ON K1N6N5, Canada
} 


\section{Background}

Malaria remains the most deadly of all vector-borne diseases, infecting an estimated 200 million people and causing over 550,000 deaths globally every year [1]. Approximately $90 \%$ of all malaria deaths occur in Africa, where an estimated $78 \%$ of deaths are in children under the age of five [1]. Malaria parasites are transmitted exclusively by Anopheles mosquitoes [2], spurring decades of research not only on the parasite, but also on its widespread vector. The spatial variation in vector-borne disease distributions such as malaria can likely be attributed to variations in environmental conditions (e.g. land cover, temperature, precipitation) and human population distribution that disease vectors depend on for survival [3]. Ecological niche models (ENMs) correlate these environmental factors with georeferenced occurrences of Anopheles mosquitoes to improve detection of spatial and temporal variation in biophysical determinants of these malaria vectors, often at a countrywide or even continent-wide scale [4-8]. Although these modelling methods are rapidly advancing, they have yet to incorporate anti-malarial intervention data, though such approaches have been suggested and encouraged [2]. Anti-malarial controls are not only time-intensive but also financially burdensome, with $\$ 5.1$ billion US planned but $\$ 2.7$ billion US expended in 2013 [1]. The synthesis of species distribution modelling (or ecological niche modelling) and large-scale mosquito control methods can offer a new perspective on the potential effects of antimalarial controls [4].

Insecticide-treated nets (ITNs) are an excellent example of such vector control methods working on a large scale. The percent reduction in malaria deaths in children under the age of five was estimated in 43 sub-Saharan African countries from 2001 to 2010, compared to a baseline in 2000. Some countries reported no reduction by ITN scale-ups, while others reported up to a $26 \%$ decrease in malaria deaths in children under the age of five [9]. In 2014, a record number of ITNs were distributed to malaria-endemic African countries, with the total number of ITNs delivered to Sub-Saharan Africa reaching 427 million [1]. Within the United Republic of Tanzania alone (hereafter Tanzania), an extensive ITN national plan has been implemented over the past 25 years, supported by the Global Fund to Fight AIDS, Tuberculosis and Malaria (GFATM) and the USA President's Malaria Initiative [10]. Since 2004, the Tanzania National Voucher Scheme has provided subsidized ITNs to pregnant women during antenatal visits [11]. Between 2009 and 2010, a mass campaign distributed 8.7 million ITNs across the country, which were free to families with children under the age of five [12]. Despite increases in coverage, analyses of ITN scale-up impacts [9] found only a $3 \%$ reduction in malaria deaths in Tanzanian children under the age of five from 2001 to 2010. In 2011, a Universal Coverage Campaign distributed 17.6 million ITNs nationally with the goal to increase global use in the general population to $80 \%$ [10]. In addition, the GFATM set a national target for Tanzania to increase the proportion of households with at least one ITN to $90 \%$ by 2013 [13].

Numerous analyses have been conducted to determine the effects of ITN use on malaria endemicity [14-17] and on mosquito populations at local scales [18-20], but none have yet compared ITN coverage with mosquito habitat suitability to determine if coverage has been optimally allocated. Environments that favour Anopheles mosquito survival and reproduction, as well as more densely populated areas that facilitate access to hosts and accelerate malaria parasite transmission, will have higher malaria risk. Given mass ITN rollouts, is ITN ownership across Tanzania meeting targets in areas with high mosquito habitat suitability and increased malaria risk? Furthermore, if ITN distributions were optimized to target the most at-risk groups, areas with greater mosquito habitat suitability would be expected to have both greater averages and lower variances in ITN ownership rates (because coverage rates should be consistently high in malaria-endemic areas). Yet, ITN ownership relative to vector suitability remains uncertain even in regions, such as Tanzania, where national household surveys exist to permit such assessments.

Here, a species distribution model is created at $1-\mathrm{km}$ resolution of Anopheles mosquitoes across Tanzania in 2001 (before large-scale ITN distributions) and is compared with countrywide ITN ownership by 2012 (number of ITNs owned per house and proportion of houses with at least one ITN) to assess where mosquitoes were most likely to thrive and whether ITN rollouts ensured coverage of such areas. This study uses the largest collection known of Anopheles mosquito occurrence records in Tanzania available for this time period, including 400 published sources, private data collections and online IR Mapper and the Malaria Atlas Project databases. This study relates species distribution models for Anopheles mosquitoes to ITN ownership and may serve as a template for integrating Anopheles mosquito distributions and disease risk with anti-malaria interventions.

\section{Methods \\ Study area}

The study area included all of Tanzania, including the large islands in the Indian Ocean (Fig. 1). Tanzania occupies $886,100 \mathrm{~km}^{2}$ [21] and its population exceeded 45 million people by 2012 [22]. Tanzania remains one of the poorest countries in the world, with a per capita average yearly income of $\$ 630$ US as of 2013 [22]. Of all 


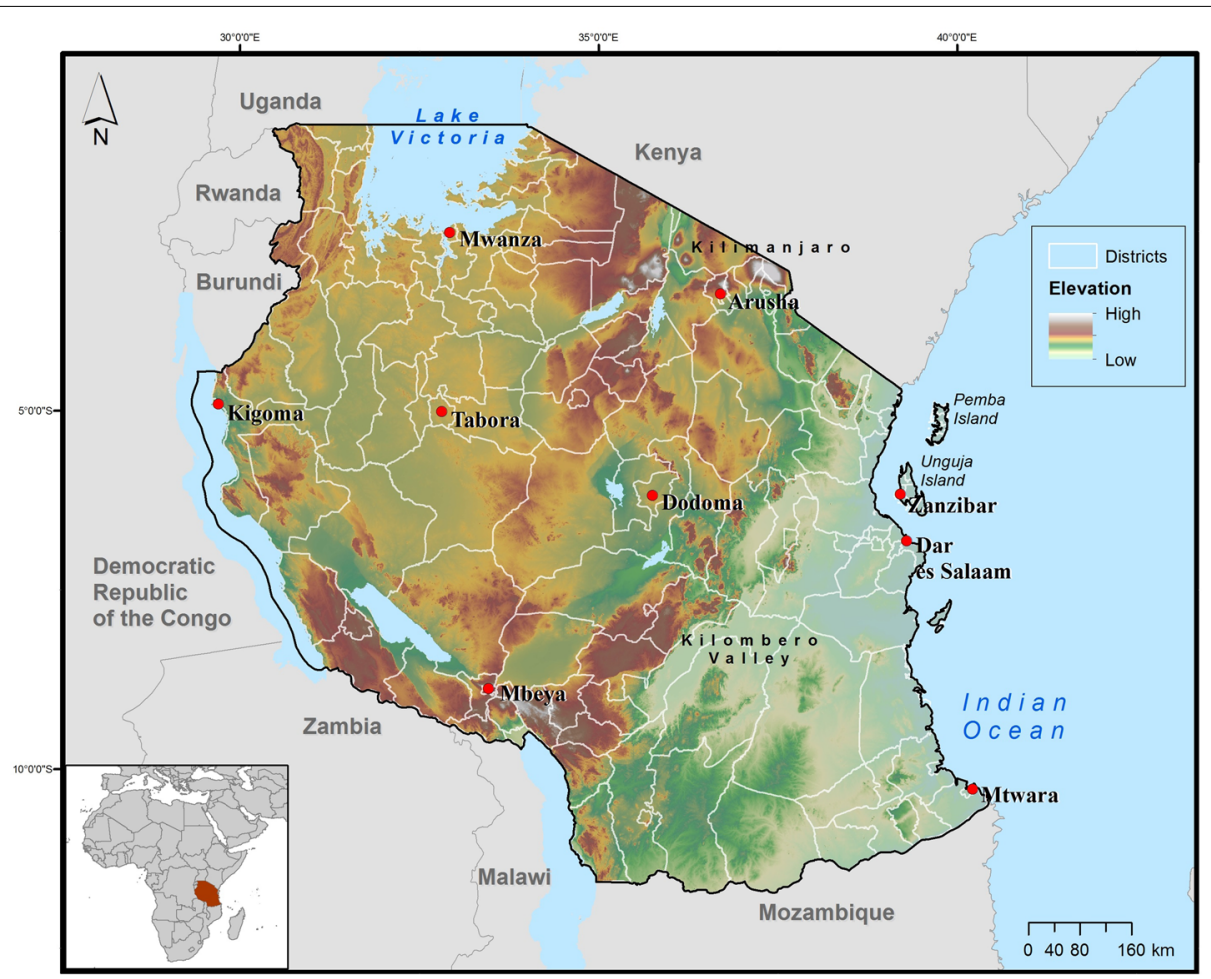

Fig. 1 United Republic of Tanzania, detailed by districts and elevation. The map shows the 148 Demographic and Health Surveys "admin 2" district boundaries, as well as elevation, several major cities, and bordering countries. The inset map details Tanzania's location within Africa

vector-borne diseases in the country, malaria still causes the highest morbidity and mortality rates [23]. Over $85 \%$ of cases are caused by Plasmodium falciparum, the most dangerous malaria parasite [24].

The country has a tropical climate with wet and dry seasons that vary due to its topography. The northern and eastern regions experience two wet seasons, with short rains from October to December and long rains from March to May. The western, central, and southern regions mainly have one wet season that lasts from October to April or May. About $80 \%$ of the population lives in rural arid regions [25] at very low population densities, with the remaining population in high-density urban centers, such as Dar es Salaam, which approaches 3133 people $/ \mathrm{km}^{2}$ [26]. Mosquito net coverage following massive ITN rollouts is not related to wealth but prioritizes pregnant women and families with children under the age of five [10].

\section{Entomological records}

The main malaria vectors in Tanzania are Anopheles arabiensis, Anopheles funestus, and Anopheles gambiae s.s. [27]. Over 400 published literature sources were reviewed for georeferenced occurrences of Anopheles mosquitoes in Tanzania. An observation for Anopheles species was recorded if collection localities were specified for any month and year of collection between 1934 and 2014. These data were supplemented by unpublished data collections (personal comm. Dr. Maureen Coetzee) and online database IR Mapper [28] and the Malaria Atlas Project database [29]. Each coordinate was verified using Google Earth (Version 7.1.2.2019, Google Inc.).

When complete, the database comprised 203 unique georeferenced observations of An. gambiae s.l. (35.5\%), An. arabiensis (32.4\%), An. funestus s.l. (16.2\%), and An. gambiae s.s. (15.9\%) across all years. Identification of mosquitoes within the An. gambiae s.l. complex relied on polymerase chain reaction or cytogenetics. The database details species, month and year of collection, citation source, village of collection, and trapping method.

\section{Niche model}

Maxent software [30], v.3.3.3k, was used to predict the relative habitat suitability of Anopheles mosquitoes across Tanzania. Maxent combines presence-only species occurrence records with environmental data to create a model that predicts areas of relative habitat suitability for a given species. Maxent uses a background dataset that consists of pseudoabsences (i.e. a random sample of non-occurrences) instead of true recorded absences. The 
output estimates relative habitat suitability and not direct occurrence probability since areas where the species was not recorded are considered less suitable relative to where the species was actually recorded. Maxent niche models with high training accuracy have been constructed for arthropod vectors, including mosquitoes [4, 31-33], ticks [34-36], tsetse flies [37-39] and sandflies [40-42]. Despite Maxent's tendency to be more conservative than other machine-learning techniques [43], it is one of the most reliable species distribution modelling methods, even with small numbers of species observations [44, 45]. However, internal training and testing accuracies evaluate the model's fit to existing observations and external tests against independent measurements or temporal environmental changes provide stronger tests of model performance [46].

Since large-scale mosquito net distributions commenced in 2004, the niche model building was narrowed to the Anopheles coordinates collected between 1999 and 2003. Many historical records for anopheline mosquitoes in the 1999-2003 period do not distinguish species within the $A n$. gambiae s.l. complex (e.g. only three geographically-unique points were recorded as An. gambiae s.s. compared to 54 recorded as $A n$. gambiae s.l.). All recorded Anopheles species were combined to build the Maxent model. The focus of this study includes any of these malaria vectors, despite niche differences among species, relative to mosquito net ownership. All eight species within the An. gambiae s.l. complex can transmit malaria parasites except Anopheles amharicus, which does not occur in Tanzania, and Anopheles quadriannulatus, which has not been recorded in the country since 1968 [47]. The absence of spatially detailed data sufficient for these models prevented species-by-species evaluation of habitat suitability for the time period relevant to national ITN distribution. In total, 56 occurrence records were thus used to build the Maxent model, substantially exceeding minimum requirements ( $>5$ observations) to produce informative predictions [44] (Fig. 2). Of these records, one was $A n$. arabiensis, seven were An. funestus, 46 were $A n$. gambiae s.l. and two were An. gambiae s.s., though more than one species was recorded at each location.

Multiple models were constructed using elevation, human population (Fig. 3), land cover, and variations of temperature and NDVI (average, minimum, and maximum). For assessment of model accuracy, occurrence records were randomly partitioned into $75 \%$ for model training and $25 \%$ for model testing. Ten replicates were run for each model, using tenfold cross-validation, each with a randomized partitioning of training and testing data, a technique that can reliably test spatial model skill for disease vector distributions [46]. The habitat suitability raster maps were then averaged to determine the relative probability of suitability per grid cell.

The model's accuracy was determined using a threshold-dependent binomial omission test and a thresholdindependent receiver operating characteristic analysis [30]. For the binomial test of omission, a fixed threshold for habitat suitability values of 0.1 was used, which is a threshold commonly set in other studies [4, 43]. For the threshold-independent analysis, model skill was evaluated using area under the receiver operating characteristic (AUC) [4, 48, 49]. AUC values approaching 1 indicate perfect discrimination between suitable and unsuitable areas for the target species, and values of 0.5 indicate performance no better than random. Each variable's unique and shared contribution to model accuracy was evaluated using jackknife procedures [30]. Each variable's relative contribution to overall model predictions was then calculated and presented as a percentage.

\section{Environmental data}

The histories of many mosquito-borne diseases, including malaria, indicate that climate is rarely the principal determinant of mosquito distributions in tropical regions and that shorter-term consequences of human activities often exert greater effects $[50,51]$. Relevant climatic factors include land surface temperature and precipitation [4, 43]. Models incorporating satellite-based land cover and/or topography as indicators of mosquito habitats can pinpoint local variations in malaria risk [52]. Human population density is a critical determinant of relative malaria risk because of density-dependent transmission of parasites among individuals in the presence of anthropophilic Anopheles vectors [43].

Measurements of environmental conditions were used at 500-1000 $\mathrm{m}$ resolution across Tanzania derived using the Moderate Resolution Imaging Spectroradiometer (MODIS) sensor on the Terra satellite. All 8-day Land Surface Temperature composites (LST; MOD11A2, $1000 \mathrm{~m}$ resolution), 12 monthly Normalized Difference Vegetation Index composites (NDVI; MOD11A3, $1000 \mathrm{~m}$ ), and land cover observations (MCD12Q1, $500 \mathrm{~m}$ ) were acquired for 2001. NDVI measures greenness and photosynthetic activity, which detects vegetation differences due to irrigation (such as rice-growing areas). Digital elevation data at 90-m resolution were obtained from the Shuttle Radar Topography Mission (SRTM; [53]) and mosaicked for the terrestrial area of Tanzania. Finally, 2001 human population distribution data were collected from the OakRidges National Laboratory LandScan database at 30 arc second $(\sim 1 \mathrm{~km})$ resolution [54] (Fig. 3).

The MODIS data were unprojected from Sinusoidal to geographic coordinate system (GCS WGS 1984 datum) 


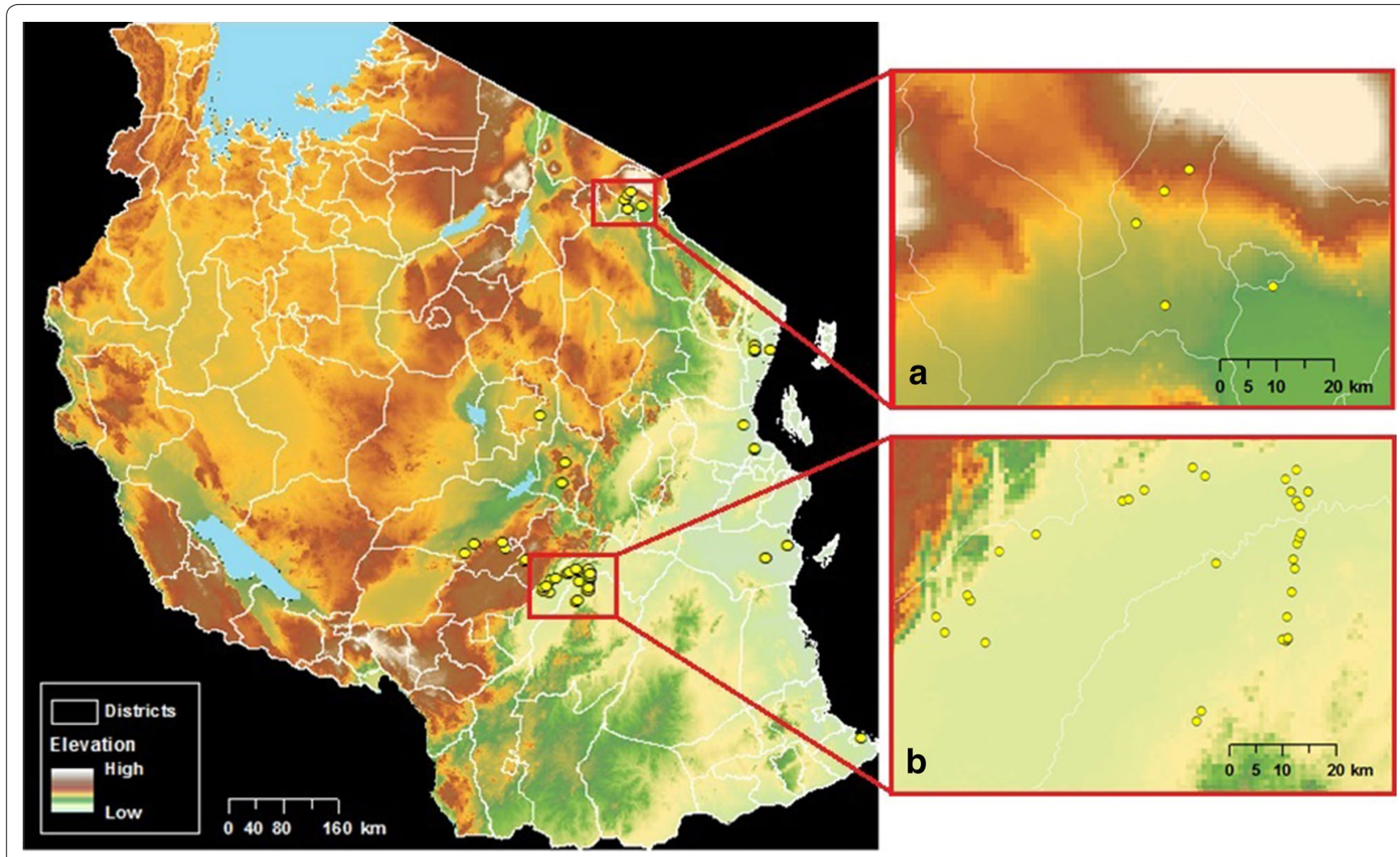

Fig. 2 Map of 56 Anopheles occurrence points used in Maxent. Insets show clustering of biased sampled points around a Mount Kilimanjaro and b Kilombero Valley

using the MODIS Reprojection Tool (MRT, v.4.0 from US Geological Survey). Human population and elevation layers were transformed to the same geographic coordinate system using ArcGIS v.10.1 (ESRI 2012, Redlands, CA). Some 8-day LST and NDVI mosaics pixels were flagged as missing or of low quality, usually due to atmospheric haze or cloud cover. Data were imported into R v.3.0.2 [55] and the raster brick function (package raster) was used to extract mean, minimum and maximum temperature and NDVI, omitting missing and low-quality pixels (see Additional file 1 for more details about the R script used for the raster brick function). Raster bricks for temperature and NDVI were then exported to ArcGIS 10.1 and all environmental layers were clipped to a land mask for Tanzania and converted to ASCII for use in Maxent.

\section{Mosquito net survey data}

This study used 2011-2012 data conducted by the AIDS Indicator Survey (AIS) throughout Tanzania, with permission from the Demographic and Health Surveys (DHS) Programme [56], funded primarily by the US Agency for International Development. AIS fieldwork was conducted between December 2011 and May 2012, implemented by Tanzania's National Bureau of Statistics. This survey provided data on number of ITNs owned per household, including long-lasting insecticide nets (LLINs). All DHS procedures and questionnaires were reviewed and approved by the ICF International Institutional Review Board. Further details are provided in each survey's final report.

To protect respondent confidentiality, the georeferenced cluster points (i.e. sample points that represented 17-18 surrounding households) were each displaced by the DHS, with shifts in latitude and longitude under set parameters [57]. Urban clusters were displaced 0-2 km and rural clusters were displaced $0-5 \mathrm{~km}$, with $1 \%$ (or every 100th point) displaced from 0 to $10 \mathrm{~km}$. Shifts in direction and distance were both random. In addition, displacement of the georeferenced clusters was restricted at the district (admin2) level for the 2011-2012 AIS survey (Fig. 1). During the randomized cluster displacement process, clusters could cross localized administrative boundaries (e.g. ward boundaries), but not a DHS region or district boundary.

\section{Mosquito net ownership layers}

For the 2011-2012 AIS survey for Tanzania, 10,496 households were selected for sampling, of which 10,040 were successfully sampled and included in the final survey results. The survey's GPS dataset comprised of 583 


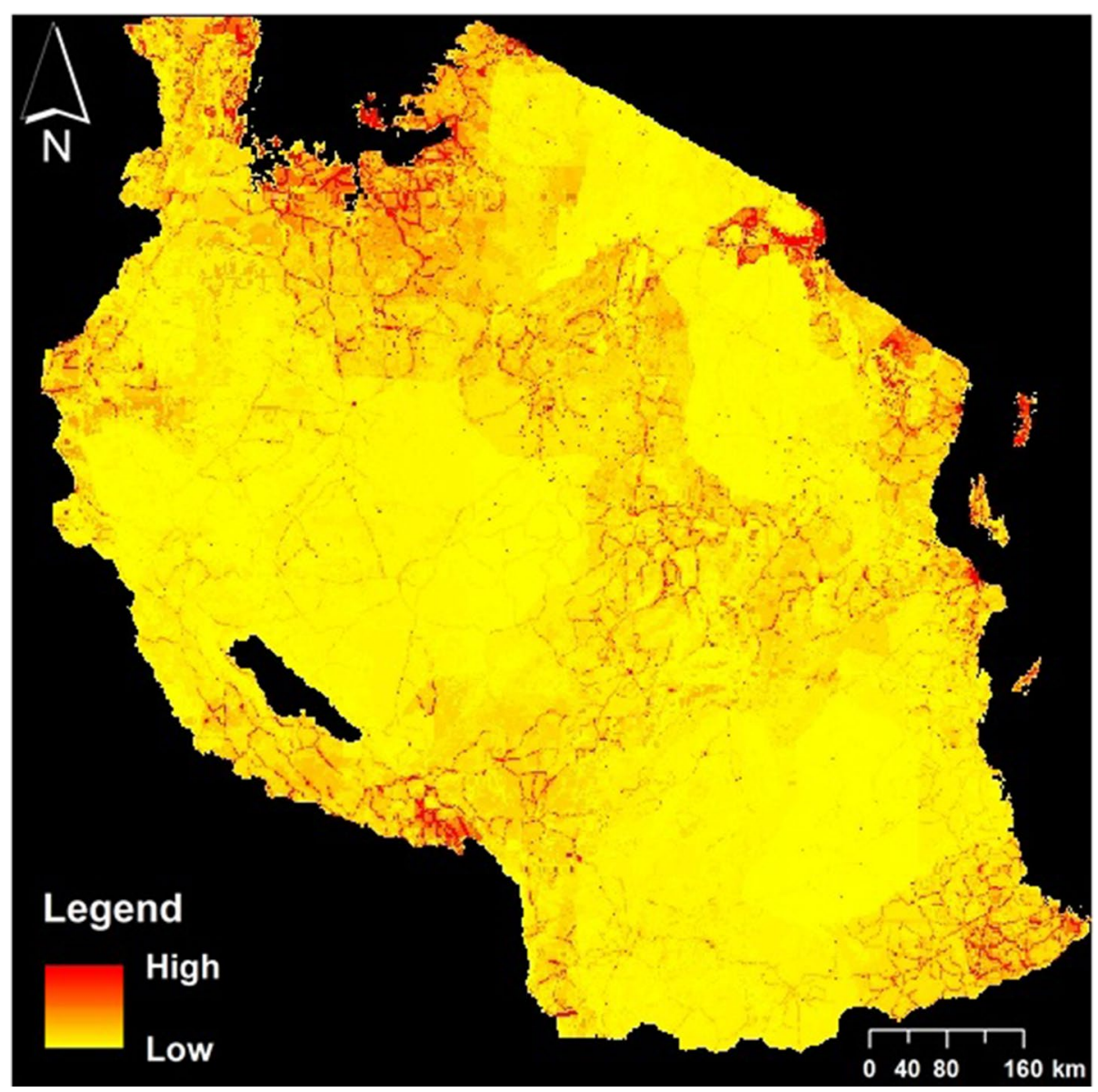

Fig. 3 Map of human population distribution from LandScan with pixel size of $\sim 1 \mathrm{~km}^{2}$

georeferenced survey cluster points, where each cluster point represented 17-18 surrounding households. Ten clusters were removed due to missing data. Of the remaining 573 clusters, 440 were in rural locations and 133 were in urban locations. Using this data set, mosquito net ownership was measured in two ways: first, the average number of mosquito nets owned per household and, second, the proportion of houses with $\geq 1$ mosquito net (Fig. 4). While both offer distinct perspectives on mosquito net ownership, the latter is particularly relevant to the stated national target of $90 \%$ of households with at least one mosquito net [13]. For each layer, the resulting calculated value from the surrounding households was assigned to their corresponding cluster coordinate.

Mosquito net ownership patterns were expected to be spatially autocorrelated and be used to estimate mosquito net ownership rates throughout unsurveyed areas of Tanzania. To test this possibility, extrapolations were attempted using kriging and inverse distance weighting techniques in ArcGIS. Predicted mosquito net ownership from extrapolated surfaces were compared to observed mosquito net ownership rates using tenfold cross validation, but model fit was very poor. Mosquito net ownership shows practically no spatial autocorrelation (measured using Moran's I on cluster points $=0.07$, $\mathrm{p}<0.05$, where Moran's I scales as the Pearson productmoment correlation between 1 and -1 , with 0 indicating no autocorrelation). Further analyses were restricted to point-based measurements.

Buffer zones were created surrounding each survey cluster, with urban clusters having a circular buffer zone with a radius of $2 \mathrm{~km}$ and rural clusters having a circular buffer zone with a radius of $5 \mathrm{~km}$. Since cluster points were randomly relocated but did not cross district boundaries, buffer zones that crossed into an adjacent district could be made more spatially precise by clipping along the district boundary. Buffer zones were consequently retained only within the district particular to each survey locality. This processing resulted in a total of 912 mosquito net polygons. The area of the buffer zone was assigned the value attributed to its cluster point for the average number of mosquito nets and the proportion 


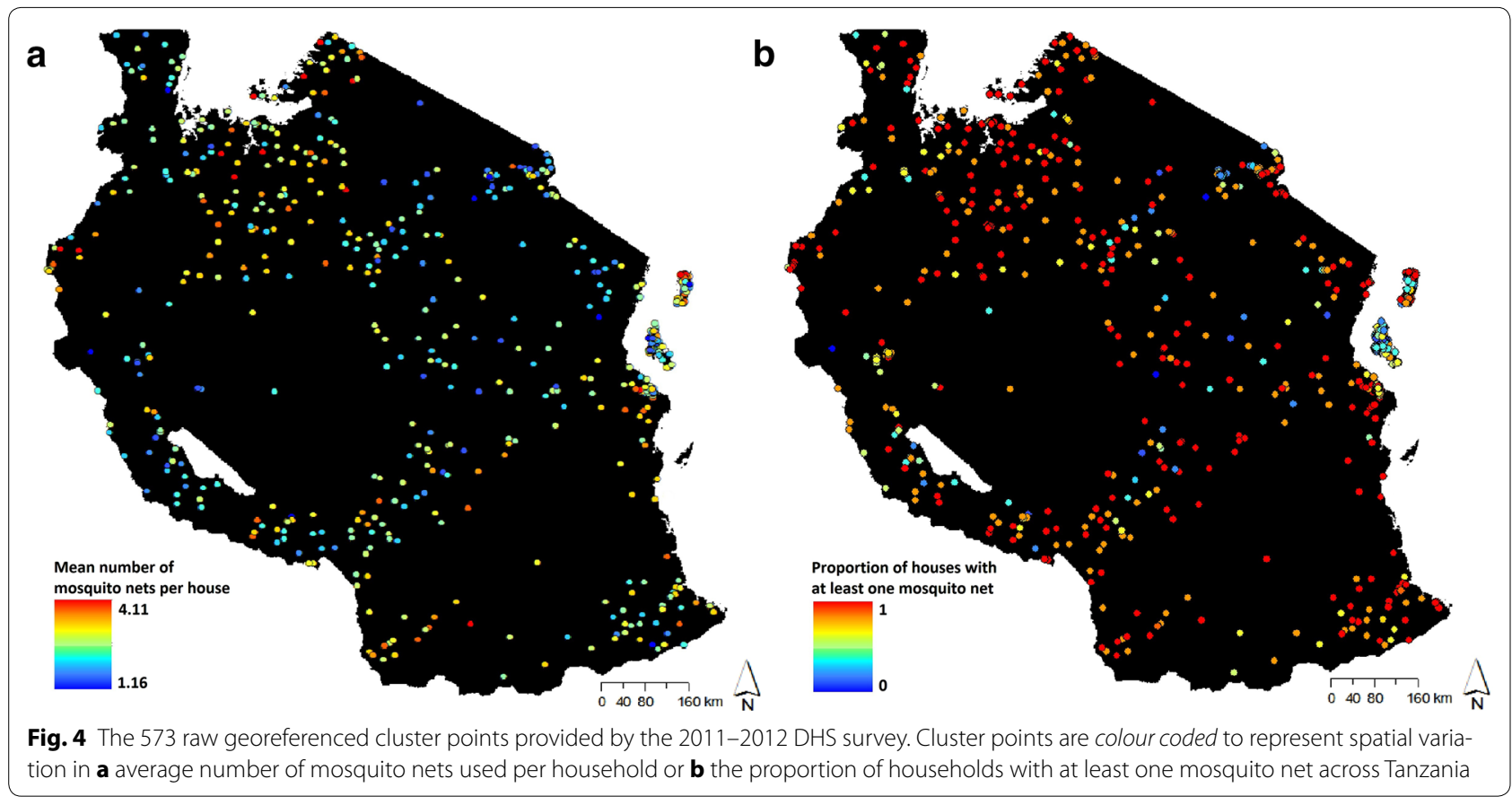

of houses with $\geq 1$ ITN. For overlapping buffer areas, the averaged value of the individual buffers was calculated (see Additional file 2 for the $\mathrm{R}$ script used to create this layer).

\section{Statistical analyses}

To relate mosquito habitat suitability to mosquito net ownership, the habitat suitability map created in Maxent and the mosquito net buffer layers created in ArcGIS 10.1 were imported into R statistical software (v.3.0.2, [55]). Since each buffer zone had either a 2 - or $5-\mathrm{km}$ radius, and had been clipped depending on proximity to district boundaries, $\mathrm{R}$ was used to calculate average habitat suitability for each unique cluster and surrounding buffer (see Additional file 3 for the R script used for this process). Ordinary least squares (OLS) regression models were then constructed to test whether Anopheles habitat suitability predicted either average numbers of mosquito nets owned per household or proportions of households with $\geq 1$ mosquito net. For each regression analysis, Levene's tests were used to test whether variances differed between household clusters that were grouped by deciles according to predicted suitability for anopheline mosquitoes. Residual spatial autocorrelation and the associated Moran's I were calculated using R. In addition, possible relationships were tested between the two measures of mosquito net ownership as a function of mosquito habitat suitability by performing quantile regression analyses in the 0.1 (the $10 \%$ of household clusters with the lowest predicted suitability for mosquitoes) and 0.9 (the $10 \%$ of household clusters with the highest predicted suitability for mosquitoes) deciles. Quantile regression is a method for providing a more complete view of possible causal relationships in ecological processes aside from the mean of the response variable distribution [58]. Using quantile regression to analyse the highest and lowest $10 \%$ of the data may reveal changes in ITN coverage within these households as mosquito habitat suitability increases that could be undetected by common regression models through the mean of the response variable. If ITN ownership is consistently high, regardless of an area's predicted suitability for anopheline mosquitoes, no trend is expected (slope $=0$ ) among quantiles. Less favourably, ITN ownership rates could increase toward areas with high suitability for these vectors, suggesting that priority for ITN distribution had been given to localities with the greatest malaria risk. Finally, ITN ownership might decline toward areas with high anopheline suitability.

\section{Results}

\section{Habitat suitability model}

Elevation, human population distribution and land cover provided stronger predictions of mosquito habitat suitability than any temperature- or NDVI-based measurements (Table 1). Model fits were comparable whether the latter measurements were omitted (AUC $=0.872$ ) or included (AUC $=0.862-0.875$ ). Elevation made the greatest contribution to the model, followed by human population distribution and land cover. Jackknife analysis attributed the maximum training gain to elevation 
Table 1 Contribution of environmental variables to Anopheles Maxent model, including and excluding variants of temperature and NDVI

\begin{tabular}{|c|c|c|c|c|}
\hline Variable & $\%$ Contribution to model & AUC with variable alone & AUC without variable & Model AUC \\
\hline \multicolumn{5}{|l|}{ Model 1} \\
\hline Elevation & 66.2 & 0.817 & 0.772 & \multirow[t]{5}{*}{0.869} \\
\hline Human population distribution & 28.2 & 0.727 & 0.773 & \\
\hline Land cover & 4.2 & 0.634 & 0.865 & \\
\hline Mean temperature & 1.2 & 0.615 & 0.874 & \\
\hline Mean NDVI & 0 & 0.593 & 0.869 & \\
\hline \multicolumn{5}{|l|}{ Model 2} \\
\hline Elevation & 65.9 & 0.817 & 0.769 & \multirow[t]{5}{*}{0.875} \\
\hline Human population distribution & 28.0 & 0.727 & 0.815 & \\
\hline Land cover & 4.3 & 0.634 & 0.870 & \\
\hline Max temperature & 1.4 & 0.546 & 0.876 & \\
\hline Max NDVI & 0.5 & 0.542 & 0.875 & \\
\hline \multicolumn{5}{|l|}{ Model 3} \\
\hline Elevation & 64.3 & 0.817 & 0.766 & \multirow[t]{5}{*}{0.862} \\
\hline Human population distribution & 27.1 & 0.727 & 0.800 & \\
\hline Land cover & 4.0 & 0.634 & 0.857 & \\
\hline Min NDVI & 2.9 & 0.606 & 0.875 & \\
\hline Min temperature & 1.6 & 0.575 & 0.862 & \\
\hline \multicolumn{5}{|l|}{ Model 4} \\
\hline Elevation & 66.5 & 0.812 & 0.764 & \multirow[t]{3}{*}{0.872} \\
\hline Human population distribution & 28 & 0.732 & 0.794 & \\
\hline Land cover & 5.5 & 0.640 & 0.876 & \\
\hline
\end{tabular}

Variants of temperature and NDVI (mean, maximum, minimum) were attempted in different models, but yielded similar minor contributions to the model. AUC, or area under the curve, values approaching 1 indicate the model's perfect discrimination between suitable and unsuitable areas for the target species, and values near 0.5 indicate performance no better than random. Model 4 was retained for analyses

(0.828), which indicates this variable contains the most information that is not present in the other variables (Fig. 5). Concentrated areas of high habitat suitability are found in low elevation areas, mainly along eastern coastlines and extending into Kilombero Valley and south toward Mozambique (Fig. 6). Areas surrounding Lake Victoria and north of Lake Malawi also contain extensive areas with high habitat suitability for anopheline mosquitoes. Dense shrub land and cropland/natural vegetation mosaic land covers were associated with higher mosquito habitat suitability. Internal accuracy of the model through the threshold-dependent binomial omission test was also high, with an omission error rate of $7.14 \%$ with a suitability threshold of 0.1 with the omission error rate remaining below $20 \%$ up to a set threshold of $0.25(\mathrm{n}=56)$. Removing elevation from Maxent models degraded model fit substantially (AUC $=0.765$ ), increasing proportional contributions of human population distribution and land cover (65 and $25.1 \%$, respectively), with minor increases in temperature and NDVI

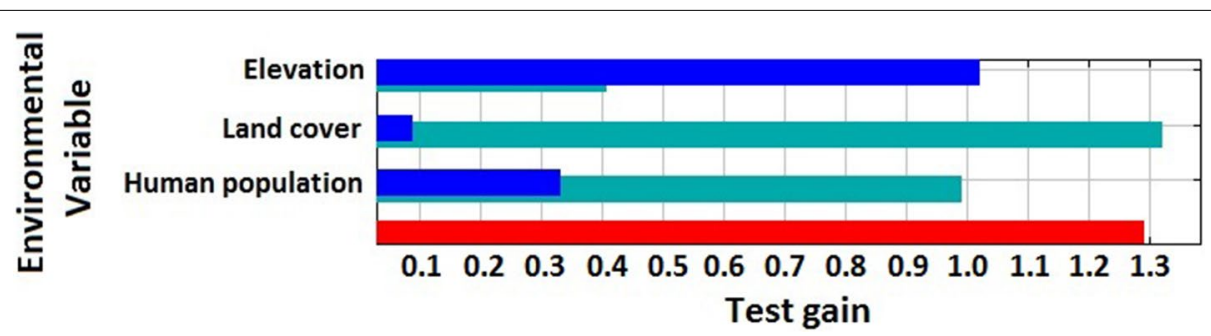

Fig. 5 Jackknife plot of testing gain for Maxent niche model building. Green bars represent test gain without the specified variable. Blue bars represent test gain with only the specified variable. The red bar represents the test gain with all variables included 


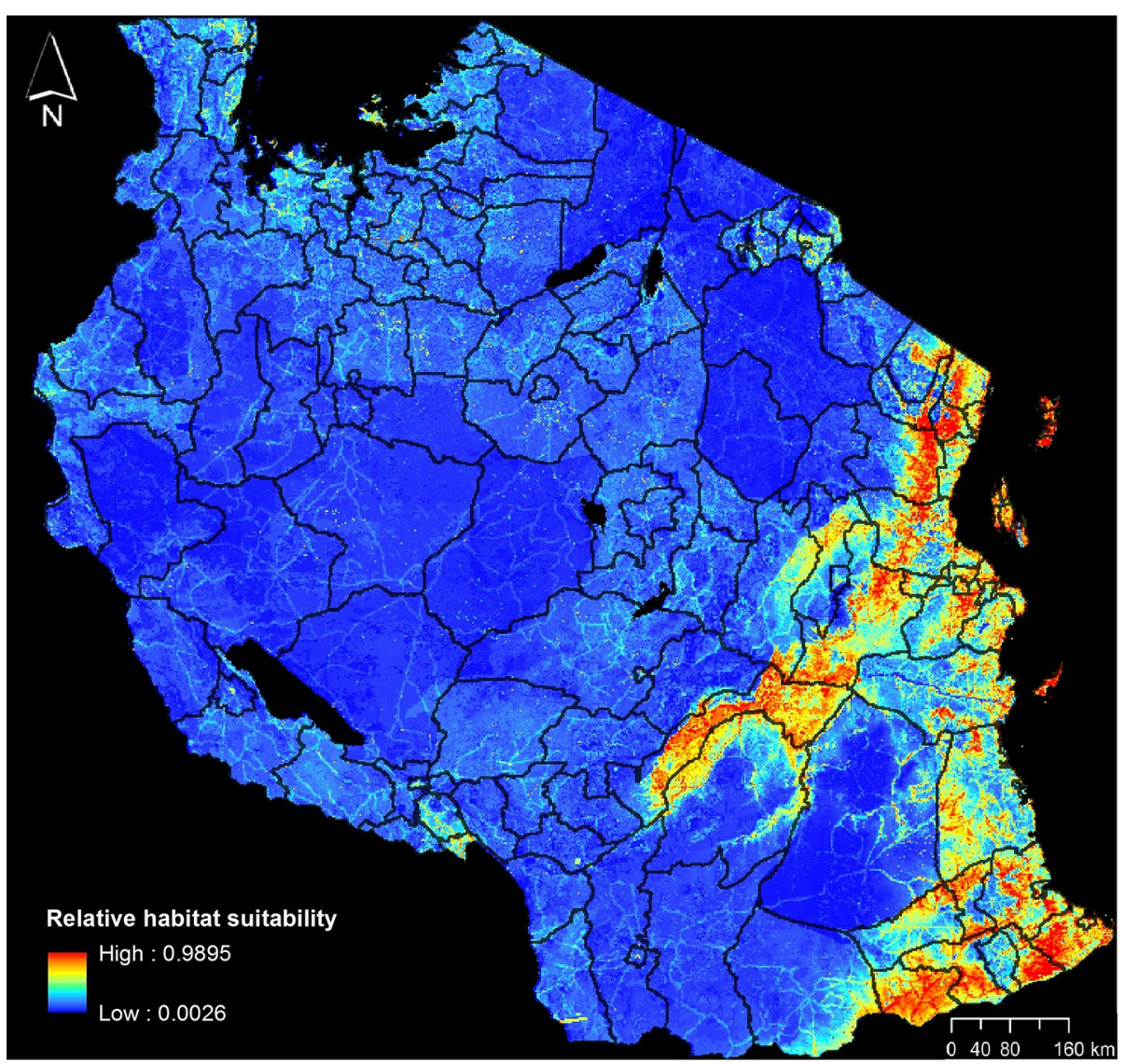

Fig. 6 Maxent output of relative Anopheles mosquito habitat suitability $(n=56)$. The habitat suitability map ranges from blue to red, with blue representing lowest predicted relative habitat suitability for Anopheles mosquitoes. The suitability map is overlaid with the "admin 2" districts of Tanzania. Predicted relative habitat suitability in many areas are coterminous with the LandScan human population data (e.g. the southeastern polygonal area of low habitat suitability corresponds substantially with the Selous Game Reserve, where human population approaches zero in most areas.)

(7.1 and $2.8 \%$, respectively). The final model excluding temperature and NDVI, but maintaining a high AUC value of 0.872 , was retained.

\section{ITN ownership and mosquito habitat suitability}

The relationship between the average number of mosquito nets owned per house and mosquito habitat suitability was not significant ( $\mathrm{p}>0.05$, Fig. 7a). Average mosquito net ownership per house did not significantly differ between rural (2.485 mosquito nets) and urban (2.491 mosquito nets) clusters $(\mathrm{t}=0.13, \mathrm{p}=0.8967)$. Variance did not significantly differ among deciles of habitat suitability $(\mathrm{F}=0.7821, \mathrm{p}=0.6331$; Fig. $7 \mathrm{~b})$. There is no relationship between mean numbers of ITNs owned per household and predicted habitat suitability among households in areas among highest or lowest mosquito habitat suitability (the top or bottom deciles, or $10 \%$, of clusters, respectively) ( $p>0.05$; Fig. 7a).

There is a slight tendency for the proportion of households with at least one mosquito net to decrease toward areas with higher mosquito habitat suitability $\left(\mathrm{R}^{2}=0.051, \mathrm{p}<1 \times 10^{-6}\right.$; Fig. $\left.7 \mathrm{c}\right)$. Overall, $93.41 \%$ of surveyed households had at least one mosquito net (93.51\% in rural households and $93.05 \%$ in urban households). Variance in ITN ownership rates across surveyed households with at least one mosquito net did differ significantly between deciles of habitat suitability $(\mathrm{F}=3.0037$, $p=0.0015$; Fig. $7 d$ ). While the 0.9 quantile of the proportion of households with at least one mosquito net showed no significant trend relative to mosquito habitat suitability, the 0.1 quantile of the proportion of households with at least one mosquito net significantly decreased as mosquito habitat suitability increased $(\beta=-0.0753$, $\mathrm{t}=-3.38, \mathrm{p}<0.001$; Fig. 7c).

\section{Discussion}

The World Health Organization (WHO) estimates 50\% of African households had access to $\geq 1$ ITN by 2010 but only $\sim 3 \%$ in 2004 [1]. This study's analysis of the DHS survey data across Tanzania from 2011 to 2012 suggests 

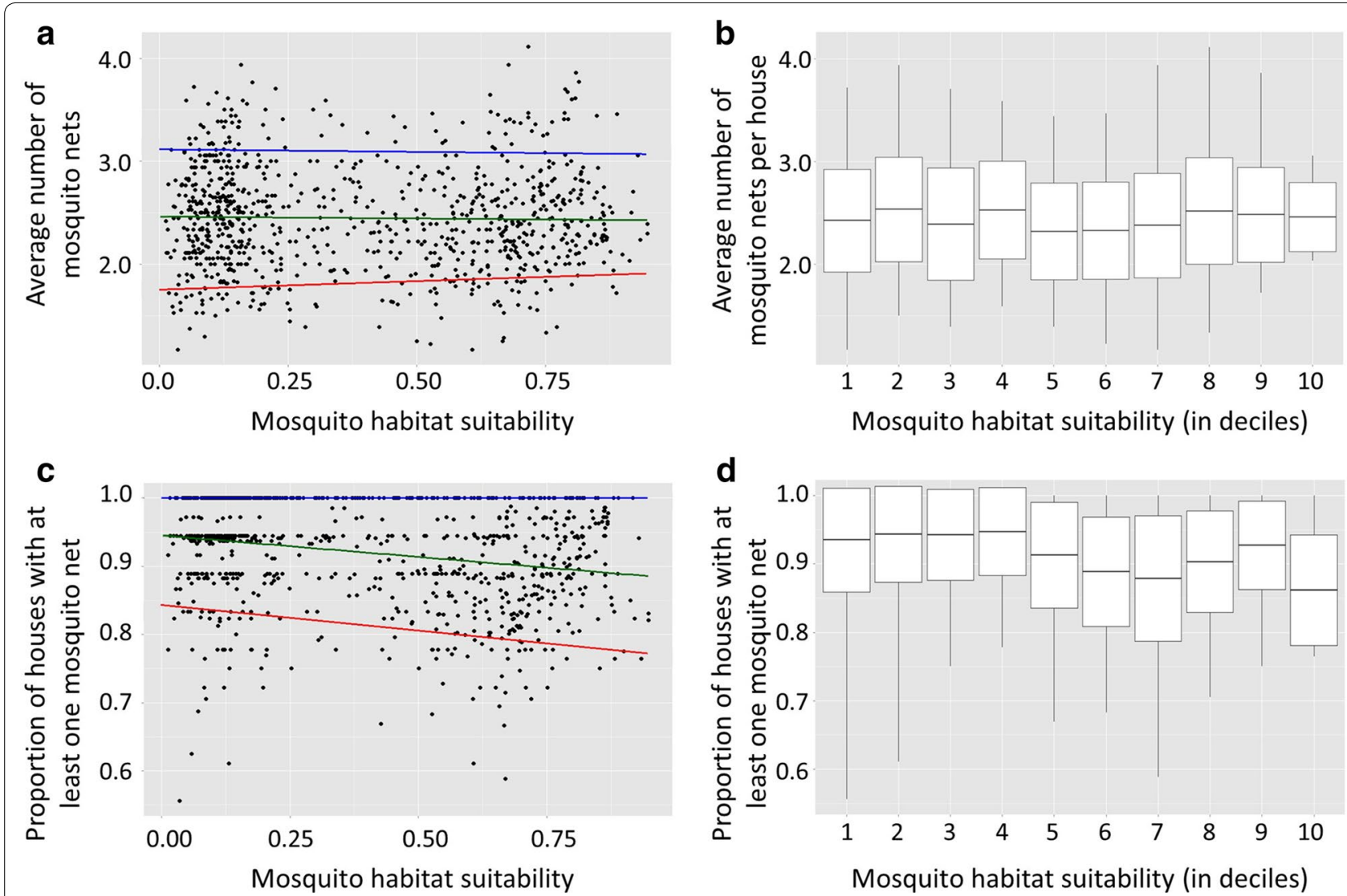

Fig. 7 Scatterplots and corresponding boxplots of mosquito net use as a function of mosquito habitat suitability. a Scatterplot of the average number of mosquito nets per house as a function of relative Anopheles habitat suitability with quantile regression and ordinary least squares (OLS) regression lines shown. The 0.9 quantile line is shown in blue, the ordinary least squares regression line is shown in green, and the 0.1 quantile line is shown in red. $\mathbf{b}$ Boxplot of mean and standard deviation of average number of mosquito nets used per house as a function of Anopheles relative habitat suitability, where habitat suitability is divided into deciles (e.g. 1 corresponds to lowest $10 \%$, etc.). Boxplot whiskers extend to the maximum and minimum values. c Scatterplot of the proportion of houses with mosquito nets as a function of relative Anopheles habitat suitability (i.e. houses with $\geq 1$ mosquito net were assigned a value of 1 ; otherwise, 0 ), with quantile regression and ordinary least squares (OLS) regression lines shown. The 0.9 quantile line is shown in blue, the ordinary least squares regression line is shown in green, and the 0.1 quantile line is shown in red. $\mathbf{d}$ Boxplot of mean and standard deviation of the proportion of houses with mosquito nets as a function of relative Anopheles habitat suitability, where habitat suitability is divided into deciles

more optimistic results, with $>93 \%$ of Tanzanian households having one or more nets, meeting the $90 \%$ national target set for 2013 [13]. There is no difference in ITN ownership rates between rural and urban households. However, the effectiveness of ITN distributions varies among communities, many of which have much lower rates of ITN ownership than national averages. Among such communities, the proportion of households with at least one mosquito net is inversely related to mosquito habitat suitability. There is a clear opportunity to improve ITN effectiveness at a national scale by targeting supplemental net distributions in areas where current ownership rates are low but mosquito habitat suitability peaks. The unexpected decrease in ITN presence in some surveyed areas where malaria risk is high likely contributes to persistent, high malaria morbidity and mortality (20,900 deaths in 2013 [59]). Other factors clearly contribute to malaria persistence, including increasing insecticide resistance among Anopheles mosquitoes in Tanzania [60-62].

It is imperative that ITNs reach households where mosquito habitat suitability, and thus malaria risk, is highest. Areas with higher mosquito habitat suitability were expected to have the most comprehensive ITN coverage and lowest variance in the number and proportion of households with one or more ITNs, but the opposite trends were observed. In areas with the highest anopheline habitat suitability, proportional ITN presence in households declines below both the national targets for ITN coverage $(90 \%)$ and the more modest ( $\geq 80 \%)$ 
international targets (77 \% of households have $\geq 1$ ITN; Fig. 7c). In other words, the proportion of households reporting no ITN ownership increases in areas where mosquito habitat suitability is highest. This gap in ITN ownership is consequential from a human health perspective but is not detectable using models describing the response variable's central tendency. Coarse resolution analyses have revealed similarly uneven mosquito net use relative to malaria endemicity among 23 African countries [14]. If internal transportation networks through which ITNs and supporting programmes are delivered (e.g. road access) caused local variability in ITN ownership rates, those patterns should have shown strong autocorrelation. Causes of variation in ITN ownership and use need investigation. These include beliefs in malaria risk, trust in health workers distributing nets, perceived benefits of the nets, education, number of children under five in the household, and availability and use of other vector control measures [63].

This study's prediction of mosquito habitat suitability relates strongly to georeferenced malaria cases and to independent distribution models of Anopheles mosquito species in Tanzania. The binomial test of omission was repeated with the fixed threshold of 0.1 to test the model against independently-collected data on unique georeferenced records of Plasmodium falciparum across Tanzania from the MAP website. This study's prediction of mosquito habitat suitability corresponded strongly to malaria records from 1985 to 2008 (model omission rate $=0.1246$ for 568 geographically-unique $P$. falciparum records) as well as those specifically within the 1999-2003 period used in this study (omission rate $=0.1321$ for 107 P. falciparum records). Model prediction (based on the fixed threshold of 0.1 ) was qualitatively consistent with the predicted occurrence maps for An. gambiae, An. arabiensis, An. funestus, and An. gambiae s.s. developed independently [64]. However, this study's model predicted a narrower distribution than Malaria Atlas Project (MAP) predictions, which likely reflects differences in the time period considered and that MAP models are intended for continental applications and have less within-country detail. This study's predictions for An. gambiae s.l. distributions were also constructed using high resolution, regional satellite data and more extensive vector observation data, which may reveal more subtle variation in relative suitability in Anopheles habitats. Although this study's Anopheles training records excluded the Lake Victoria region, this study's model correctly predicted high relative habitat suitability there. This region is a malaria hotspot [65], with malaria steadily spreading to higher altitudes and aggravated by climate variability and poverty [66].
The distribution of anopheline mosquitoes and malaria is strongly influenced by land cover $[6,67]$ and human population distributions $[43,68]$ in addition to climatic and topographical factors [43, 69-71]. Elevation also limits Anopheles distributions [4, 43, 72] and its omission from ecological niche models can severely degrade both model fit and prediction accuracy [73]. In this study, mosquito habitat suitability increased particularly in cropland/natural land cover, where irrigation and water pooling are common, and in dense shrublands. Land features, such as soil type, are not directly measured in these satellite land cover data but may help explain such findings through differences in soil water holding capacity that can alter mosquito breeding success [67]. Temperature and NDVI explained little unique variation in habitat suitability, though both can relate to mosquito distributions under some circumstances $[4,8,72,74]$. Temperature shows relatively little spatial variation across Tanzania except along elevation gradients [75], which are more directly measured by the elevation variable. NDVI relates to vegetation greenness, productivity, and moisture availability, which are also strongly related to mosquito reproduction [76]. However, satellite-based land cover measurements provide similar measurements that may more accurately detect the influences of vegetation on mosquito distributions. Furthermore, highly productive vegetation (i.e. areas with high NDVI) can be found in many regions of Tanzania, including at high elevations that are too cold to permit malaria transmission, eroding the capacity for such measures to discriminate between suitable and unsuitable mosquito habitats at this scale of analysis.

While this study's dataset includes the most comprehensive collection of spatially-unique georeferenced Anopheles records for this region to date, systematic, randomized, broad-scale sampling that identifies species in the An. gambiae complex would be extremely valuable. Presence-only species observations assembled from an array of sources that differ in sampling effort and geographical focus could bias models toward areas with easier access (e.g. roadsides, proximity to research centres). In addition, lack of species-by-species evaluation for the historical period forced the combination of Anopheles species. By combining the occurrence records across the An. gambiae s.l. complex, the applicability of model predictions to particular species is limited (e.g. An. arabiensis inhabits more arid regions than An. gambiae s.s. and An. funestus, better tolerating decreasing precipitation and interruptions in rainfall patterns in certain regions of Tanzania [8]), but the relationship with malaria risk remains very strong. Systematic sampling at the species level, including observations of absence, is needed. ITN 
ownership and subsequent use may differ depending on which Anopheles species is locally common (e.g. An. arabiensis is more common outdoors [72], rendering it less affected by ITNs than An. gambiae s.s. and An. funestus [77]). Systematic, repeated sampling of Anopheles mosquitoes across Tanzania could significantly improve the capacity to target interventions, and would provide a stronger basis for predicting how distributions of these vectors would change through time [78].

\section{Conclusions}

Insecticide-treated nets have been broadly distributed across Tanzania but ownership is uneven and declines in areas where anopheline mosquitoes are concentrated. The combination of species distribution models, drawing on high-resolution satellite information, and georeferenced mosquito net data can provide useful perspective on the effectiveness of such interventions in areas where georeferenced vector and vector control data exist. These techniques are transferrable to other vector-borne disease systems. With 3.2 billion people at risk of malaria infection worldwide [1], the need to control malaria is critical. Linking ITN ownership rates to validated mosquito species distribution models can help optimize allocation of limited resources, reveal gaps in such intervention programmes, and help minimize malaria cases where risk is greatest.

\section{Additional files}

Additional file 1: R script used for raster brick function. This document contains the annotated code used to perform the raster brick function on the temperature and NDVI data in R.

Additional file 2: $\mathrm{R}$ script used to create mosquito net buffer zone layer. This document contains the annotated code used to create the layer containing the mosquito net layer, including how the buffer zones were clipped to district boundaries, and averaged for overlapping areas using $R$.

Additional file 3: $R$ script used to calculate average of habitat suitability pixels under each buffer zone. This document contains the annotated code used to calculate the average pixel value under each buffer zone (overlapping areas act as a separate zone with their own averaged value) using $R$.

\section{Abbreviations}

AIS: AIDS Indicator Survey; DHS: Demographic and Health Surveys; ENM: Ecological niche model; GFATM: Global fund to fight AIDS, tuberculosis and malaria; ITN: Insecticide-treated nets; LLIN: Long-lasting insecticide-treated nets; MAP: Malaria Atlas Project; MODIS: Moderate resolution imaging spectroradiometer; NDVI: Normalized Difference Vegetation Index; SRTM: Shuttle radar topography mission; WHO: World Health Organization.

\section{Authors' contributions}

EA and JK conceived of the study. EA performed data collection. All three authors participated in data and statistical analysis. EA drafted the manuscript and AP and JK revised it critically for important intellectual content. All authors read and approved the final manuscript.

\section{Acknowledgements}

We would like to thank Dr. Maureen Coetzee, Dr. Marianne Sinka, Dr. Rachelle Desrochers, Dr. Manisha Kulkarni, and Juan Zuloaga for data or advice with the design and data analysis. We would also like to thank the DHS staff for assistance with the DHS survey data. ESA acknowledges the Natural Sciences and Engineering Research Council of Canada (NSERC) and the Queen Elizabeth II Graduate Scholarship in Science and Technology for support, and JTK is grateful for funding for this research, including the NSERC Discovery Grant and University Research Chair support at the University of Ottawa.

\section{Compliance with ethical guidelines}

\section{Competing interests}

The authors declare that they have no competing interests.

Received: 5 May 2015 Accepted: 6 August 2015

Published online: 19 August 2015

\section{References}

1. WHO (2014) World Malaria Report 2014. World Health Organization, Geneva

2. Kelly-Hope LA, Hemingway J, McKenzie FE (2009) Environmental factors associated with the malaria vectors Anopheles gambiae and Anopheles funestus in Kenya. Malar J 8:268

3. Ernst KC, Adoka SO, Kowuor DO, Wilson ML, John CC (2006) Malaria hotspot areas in a highland Kenya site are consistent in epidemic and non-epidemic years and are associated with ecological factors. Malar J $5: 78$

4. Kulkarni MA, Desrochers RE, Kerr JT (2010) High resolution niche models of malaria vectors in northern Tanzania: a new capacity to predict malaria risk? PLoS One 5:e9396

5. Fuller DO, Ahumada ML, Quinones ML, Herrera S, Beier JC (2012) Nearpresent and future distribution of Anopheles albimanus in Mesoamerica and the Caribbean Basin modeled with climate and topographic data. Int J Health Geogr 11:13

6. Fuller DO, Parenti MS, Hassan AN, Beier JC (2012) Linking land cover and species distribution models to project potential ranges of malaria vectors: an example using Anopheles arabiensis in Sudan and Upper Egypt. Malar J $11: 264$

7. Tonnang HEZ, Kangalawe RYM, Yanda PZ (2010) Predicting and mapping malaria under climate change scenarios: the potential redistribution of malaria vectors in Africa. Malar J 9:111

8. Drake JM, Beier JC (2014) Ecological niche and potential distribution of Anopheles arabiensis in Africa in 2050. Malar J 13:213

9. Eisele TP, Larsen DA, Walker N, Cibulskis RE, Yukich JO, Zikusooka CM et al (2012) Estimates of child deaths prevented from malaria prevention scale-up in Africa 2001-2010. Malar J 11:93

10. West PA, Protopopoff N, Rowland MW, Kirby MJ, Oxborough RM, Mosha FW et al (2012) Evaluation of a national universal coverage campaign of long-lasting insecticidal nets in a rural district in north-west Tanzania. Malar J 11:273

11. Marchant T, Schellenberg D, Nathan R, Armstrong-Schellenberg J, Mponda $\mathrm{H}$, Jones C et al (2010) Assessment of a national voucher scheme to deliver insecticide-treated mosquito nets to pregnant women. CMAJ 182:152-156

12. Bonner K, Mwita A, McElroy PD, Omari S, Mzava A, Lengeler C et al (2011) Design, implementation and evaluation of a national campaign to distribute nine million free LLINs to children under 5 years of age in Tanzania. Malar J 10:12

13. (2011) The global fund to fight AIDS, tuberculosis and malaria: Grant scorecard (TNZ-M-13). http://portfolio.theglobalfund.org/en/Grant/ Index/TNZ-809-G11-M. Accessed 14 Feb 2015

14. Vanderelst D, Speybroeck N (2013) An adjusted bed net coverage indicator with estimations for 23 African countries. Malar J 12:457

15. Ngonghala CN, Del Valle SY, Zhao RJ, Mohammed-Awel J (2014) Quantifying the impact of decay in bed-net efficacy on malaria transmission. J Theor Biol 363:247-261 
16. Larsen DA, Hutchinson P, Bennett A, Yukich J, Anglewicz P, Keating J et al (2014) Community coverage with insecticide-treated mosquito nets and observed associations with all-cause child mortality and malaria parasite infections. Am J Trop Med Hyg 91:950-958

17. Burgert CR, Bradley SEK, Arnold F, Eckert E (2014) Improving estimates of insecticide-treated mosquito net coverage from household surveys: using geographic coordinates to account for endemicity. Malar J 13:254

18. Futami K, Dida GO, Sonye GO, Lutiali PA, Mwania MS, Wagalla S et al (2014) Impacts of insecticide treated bed nets on Anopheles gambiae s.l. populations in Mbita district and Suba district, Western Kenya. Parasit Vectors 7:63

19. Bayoh MN, Walker ED, Kosgei J, Ombok M, Olang GB, Githeko AK et al (2014) Persistently high estimates of late night, indoor exposure to malaria vectors despite high coverage of insecticide treated nets. Parasit Vectors 7:13

20. McCann RS, Ochomo E, Bayoh MN, Vulule JM, Hamel MJ, Gimnig JE et al (2014) Reemergence of Anopheles funestus as a vector of Plasmodium falciparum in western Kenya after long-term implementation of insecticidetreated bed nets. Am J Trop Med Hyg 90:597-604

21. NBS (2013) Tanzania in Figures 2012. National Bureau of Statistics, Tanzania

22. (2015) Data:Tanzania. http://data.worldbank.org/country/tanzania. Accessed 19 Feb 2015

23. Mboera LEG, Mayala BK, Kweka EJ, Mazigo HD (2011) Impact of climate change on human health and health systems in Tanzania: a review. Tanzan J Health Res 13:1-23

24. (2013) Malaria Information and Prophylaxis, by Country [T]. http://www. cdc.gov/malaria/travelers/country_table/t.html. Accessed 9 Jan 2015

25. Mtega WP, Ronald B (2013) The state of rural information and communication services in Tanzania: a meta-analysis. Int J Inf Commun Technol Res 3:64-73

26. NBS (2013) 2012 Population and Housing Census. In: National Bureau of Statistics. National Bureau of Statistics, Dar Es Salaam

27. Temu EA, Minjas JN, Coetzee M, Hunt RH, Shiff CJ (1998) The role of four anopheline species (Diptera: Culicidae) in malaria transmission in coastal Tanzania. Trans R Soc Trop Med Hyg 92:152-158

28. IR Mapper. http://www.irmapper.com/. Accessed 15 Feb 2015

29. The Malaria Atlas Project website. http://www.map.ox.ac.uk. Accessed 4 Nov 2014

30. Phillips SJ, Anderson RP, Schapire RE (2006) Maximum entropy modeling of species geographic distributions. Ecol Model 190:231-259

31. Foley DH, Klein TA, Kim HC, Brown T, Wilkerson RC, Rueda LM (2010) Validation of ecological niche models for potential malaria vectors in the Republic of Korea. J Am Mosq Control Assoc 26:210-213

32. Rochlin I, Ninivaggi DV, Hutchinson ML, Farajollahi A (2013) Climate change and range expansion of the Asian Tiger Mosquito (Aedes albopictus) in Northeastern USA: Implications for public health practitioners. PLoS One 8:e60874

33. Mughini-Gras L, Mulatti P, Severini F, Boccolini D, Romi R, Bongiorno G et al (2014) Ecological niche modelling of potential West Nile Virus Vector mosquito species and their geographical association with equine epizootics in Italy. Ecohealth 11:120-132

34. Feria-Arroyo TP, Castro-Arellano I, Gordillo-Perez G, Cavazos AL, VargasSandoval M, Grover A et al (2014) Implications of climate change on the distribution of the tick vector Ixodes scapularis and risk for Lyme disease in the Texas-Mexico transboundary region. Parasit Vectors 7:199

35. Giles JR, Peterson AT, Busch JD, Olafson PU, Scoles GA, Davey RB et al (2014) Invasive potential of cattle fever ticks in the southern United States. Parasit Vectors 7:189

36. Porretta D, Mastrantonio V, Amendolia S, Gaiarsa S, Epis S, Genchi C et al (2013) Effects of global changes on the climatic niche of the tick /xodes ricinus inferred by species distribution modelling. Parasit Vectors 6:1-8

37. Dicko AH, Lancelot R, Seck MT, Guerrini L, Sall B, Lo M et al (2014) Using species distribution models to optimize vector control in the framework of the tsetse eradication campaign in Senegal. Proc Natl Acad Sci USA 111:10149-10154

38. Matawa F, Murwira KS, Shereni W (2013) Modelling the distribution of suitable Glossina Spp. habitat in the North Western parts of Zimbabwe using remote sensing and climate data. Geoinform Geostast Overv 1-9

39. Bouyer F, Seck MT, Dicko AH, Sall B, Lo M, Vreysen MJB et al (2014) Ex-ante benefit-cost analysis of the elimination of a Glossina palpalis gambiensis population in the Niayes of Senegal. PLoS Negl Trop Dis 8:e3112
40. Samy AM, Campbell LP, Peterson AT (2014) Leishmaniasis transmission: distribution and coarse-resolution ecology of two vectors and two parasites in Egypt. Rev Da Soc Bras De Med Trop 47:57-62

41. Fischer D, Moeller P, Thomas SM, Naucke TJ, Beierkuhnlein C (2011) Combining climatic projections and dispersal ability: a method for estimating the responses of sandfly vector species to climate change. PLoS Negl Trop Dis 5:e1407

42. Colacicco-Mayhugh MG, Masuoka PM, Grieco JP (2010) Ecological niche model of Phlebotomus alexandri and P. papatasi (Diptera: Psychodidae) in the Middle East. Int J Health Geogr 9:2

43. Moffett A, Shackelford N, Sarkar S (2007) Malaria in Africa: Vector species' niche models and relative risk maps. PLoS One 2:e824

44. Pearson RG, Raxworthy CJ, Nakamura M, Peterson AT (2007) Predicting species distributions from small numbers of occurrence records: a test case using cryptic geckos in Madagascar. J Biogeogr 34:102-117

45. Hernandez PA, Franke I, Herzog SK, Pacheco V, Paniagua L, Quintana HL et al (2008) Predicting species distributions in poorly-studied landscapes. Biodivers Conserv 17:1353-1366

46. Acheson ES, Kerr JT (2015) Looking forward by looking back: Using historical calibration to improve forecasts of human disease vector distributions. Vector Borne Zoonotic Dis 15:173-183

47. Coetzee M, Hunt RH, Wilkerson R, della Torre A, Coulibaly MB, Besansky NJ (2013) Anopheles coluzzii and Anopheles amharicus, new members of the Anopheles gambiae complex. Zootaxa 3619:246-274

48. Moo-Llanes D, Ibarra-Cerdena CN, Rebollar-Tellez EA, Ibanez-Bernal S, Gonzalez C, Ramsey JM (2013) Current and future niche of North and Central American sand flies (Diptera: Psychodidae) in climate change scenarios. PLoS Negl Trop Dis 7:e2421

49. Estrada-Pena A, Venzal JM (2007) Climate niches of tick species in the Mediterranean region: Modeling of occurrence data, distributional constraints, and impact of climate change. J Med Entomol 44:1130-1138

50. Reiter P (2001) Climate change and mosquito-borne disease. Environ Health Perspect 109:141-161

51. Rogers DJ, Randolph SE (2000) The global spread of malaria in a future, warmer world. Science 289:1763-1766

52. Kerr J, Kulkarni M, Algar A (2011) Integrating theory and predictive modeling for conservation research. In: Drew C, Wiersma YF, Huettmann $\mathrm{F}$ (eds) Predictive Species and Habitat Modeling in Landscape Ecology. Springer, New York, pp 9-28

53. (2006) SRTM 90 m Digital Elevation Database v4.1. http://www.cgiar-csi. org/data/srtm-90m-digital-elevation-database-v4-1. Accessed 25 Oct 2014

54. (2001) LandScan. http://web.ornl.gov/sci/landscan/. Accessed 6 Apr 2014

55. (2013) The R Project for Statistical Computing. http://www.r-project.org/. Accessed 2 Nov 2013

56. (2012) The Demographic and Health Surveys Programme. http://www. dhsprogram.com/. Accessed 5 Jan 2014

57. Perez-Heydrich C, Warren JL, Burgert CR, Emch ME (2013) Guidelines on the Use of DHS GPS data: DHS Spatial Analyst Reports 8. In: Moore S (ed) USAID, Calverton

58. Cade BS, Noon BR (2003) A gentle introduction to quantile regression for ecologists. Front Ecol Environ 1:412-420

59. (2015) United Republic of Tanzania:WHO statistical profile. http://www. who.int/gho/countries/tza.pdf?ua=1. Accessed 16 Feb 2015

60. Matowo J, Jones CM, Kabula B, Ranson H, Steen K, Mosha F et al (2014) Genetic basis of pyrethroid resistance in a population of Anopheles arabiensis, the primary malaria vector in Lower Moshi, north-eastern Tanzania. Parasit Vectors 7:274

61. Protopopoff N, Matowo J, Malima R, Kavishe R, Kaaya R, Wright A et al (2013) High level of resistance in the mosquito Anopheles gambiae to pyrethroid insecticides and reduced susceptibility to bendiocarb in north-western Tanzania. Malar J 12:149

62. Nkya TE, Akhouayri I, Poupardin R, Batengana B, Mosha F, Magesa S et al (2014) Insecticide resistance mechanisms associated with different environments in the malaria vector Anopheles gambiae: a case study in Tanzania. Malar J 13:28

63. Graves PM, Ngondi JM, Hwang J, Getachew A, Gebre T, Mosher AW et al (2011) Factors associated with mosquito net use by individuals in households owning nets in Ethiopia. Malar J 10:354 
64. (2010) Tanzania (United Republic of). http://www.map.ox.ac.uk/explore/ countries/TZA/\#!mosquito-vectors. Accessed 4 Nov 2014

65. Gething PW, Patil AP, Smith DL, Guerra CA, Elyazar IRF, Johnston GL et al (2011) A new world malaria map: Plasmodium falciparum endemicity in 2010. Malar J 10:378

66. Wandiga SO, Opondo M, Olago D, Githeko A, Githui F, Marshall M et al (2010) Vulnerability to epidemic malaria in the highlands of Lake Victoria basin: the role of climate change/variability, hydrology and socio-economic factors. Clim Change 99:473-497

67. Foley DH, Klein TA, Kim HC, Sames WJ, Wilkerson RC, Rueda LM (2009) Geographic distribution and ecology of potential malaria vectors in the Republic of Korea. J Med Entomol 46:680-692

68. Ayala D, Costantini C, Ose K, Kamdem GC, Antonio-Nkondjio C, Agbor J et al (2009) Habitat suitability and ecological niche profile of major malaria vectors in Cameroon. Malar J 8:307

69. Stefani A, Dusfour I, Cruz MCB, Dessay N, Galardo AKR, Galardo CD et al (2013) Land cover, land use and malaria in the Amazon: a systematic literature review of studies using remotely sensed data. Malar J 12:192

70. Ermert V, Fink AH, Morse AP, Paeth H (2012) The impact of regional climate change on malaria risk due to greenhouse forcing and land-use changes in tropical Africa. Environ Health Perspect 120:77-84

71. Mutuku FM, Bayoh MN, Hightower AW, Vulule JM, Gimnig JE, Mueke JM et al (2009) A supervised land cover classification of a western Kenya lowland endemic for human malaria: associations of land cover with larval Anopheles habitats. Int J Health Geogr 8:19
72. Sinka ME, Bangs MJ, Manguin S, Coetzee M, Mbogo CM, Hemingway J et al (2010) The dominant Anopheles vectors of human malaria in Africa, Europe and the Middle East: occurrence data, distribution maps and bionomic precis. Parasit Vectors 3:117

73. Sweeney AW, Beebe NW, Cooper RD, Bauer JT, Peterson AT (2006) Environmental factors associated with distribution and range limits of malaria vector Anopheles farauti in Australia. J Med Entomol 43:1068-1075

74. Obsomer V, Defourny P, Coosemans M (2012) Predicted distribution of major malaria vectors belonging to the Anopheles dirus complex in Asia: Ecological niche and environmental influences. PLoS One 7:e50475

75. Goyal MR, Harmsen EW (2014) Evapotranspiration: Temperature versus elevation relationships. In: Evapotranspiration: Principles and Applications for Water Management, vol 1. Apple Academic Press, Inc., Oakville

76. Yamana TK, Eltahir EAB (2013) Incorporating the effects of humidity in a mechanistic model of Anopheles gambiae mosquito population dynamics in the Sahel region of Africa. Parasit Vectors 6:235

77. Kitau J, Oxborough RM, Tungu PK, Matowo J, Malima RC, Magesa SM et al (2012) Species shifts in the Anopheles gambiae complex: Do LLINs successfully control Anopheles arabiensis? PLoS One 7:e31481

78. Obsomer V, Titeux N, Vancustem C, Duveiller G, Pekel J, Connor S et al (2013) From Anopheles to spatial surveillance: a roadmap through a multidisciplinary challenge. In: Manguin S, CC BY (eds) Anopheles mosquitoes: New insights into malaria vectors

\section{Submit your next manuscript to BioMed Central and take full advantage of:}

- Convenient online submission

- Thorough peer review

- No space constraints or color figure charges

- Immediate publication on acceptance

- Inclusion in PubMed, CAS, Scopus and Google Scholar

- Research which is freely available for redistribution

Submit your manuscript at www.biomedcentral.com/submit
C Biomed Central 